\title{
ON EXCEPTIONAL VALUES OF ENTIRE AND MEROMORPHIC FUNCTIONS
}

\author{
SHRI KRISHNA SINGH
}

(Received February 10,1961)

1. Let $F(z)$ be a meromorphic function and let $T(r, F)$ be its Nevanlinna characteristic function. Let $N(r, a)=N(r, F-a) ; N(r, F)=N(r, \infty)$ have the usual meaning in the Nevanlinna theory.

Define

$$
\begin{aligned}
& \delta(a)=1-\limsup _{r \rightarrow \infty} \frac{N(r, a)}{T(r, F)}, \\
& \Delta(a)=1-\liminf _{r \rightarrow \infty} \frac{N(r, a)}{T(r, F)} .
\end{aligned}
$$

If $\delta(a)>0$ we say that $a$ is an exceptional value for $F(z)$ in the sense of Nevanlinna (e. v. N); and if $\Delta(a)>0$ we call $a$ as an e. v.V (exceptional value in the sense of Valiron).

2. Let $f(z)$ be an entire function and let

$$
\mu(r ; f)=\mu(r)=\operatorname{Min}_{|z| \neq r}|f(z)| .
$$

It is clear that if 0 is an asymptotic value for $f(z)$ then $\mu(r) \rightarrow 0$ as $r \rightarrow \infty$. We show that the converse is not true. We prove:

THEOREM 1. For an entire function $f(z)$, the minimurn modulus $\mu(r)$ tending to zero does not imply that 0 is an asymptotic value.

LEMMA If 0 is an e. $v . N$ for the entire function $f(z)$ then $\mu(r) \rightarrow 0$ as $r \rightarrow \infty$.

PROOF. In the terminology of Nevanlinna

$$
m\left(r, \frac{1}{f}\right)=m(r, 0)=\frac{1}{2 \pi} \int_{0}^{2 \pi} \log ^{+}\left|\frac{1}{f\left(r e^{i \theta}\right)}\right| d \theta .
$$

Hence

$$
m(r, 0) \leqq \log ^{+} \frac{1}{\mu(r)}
$$

But

$$
\lim _{r \rightarrow \infty} \inf \frac{m(r, 0)}{T(r, f)}>0 \text {, }
$$


because 0 is an e. v. $\mathrm{N}$, so

$$
T(r, f) \leqq A \log ^{+} \frac{1}{\mu(r)} \quad \text { for } r \geqq r_{0}
$$

and the lemma follows because

$$
T(r, f) \rightarrow \infty \text { as } r \rightarrow \infty
$$

To complete the proof of Theorem 1, let

$$
\begin{aligned}
& f_{1}(z)=\prod_{\nu=1}^{\infty}\left(1+\frac{z^{2}}{\nu^{4 / 3}}\right), \\
& f_{2}(z)=\prod_{n=1}^{\infty} \phi_{n}\left[(-1)^{n} z\right]
\end{aligned}
$$

where

$$
\begin{gathered}
\phi_{n}(z)=\left(1+\frac{i z}{r_{n}}\right)^{\lambda_{n}} \exp \left(\frac{-\lambda_{n} i z_{n}}{r_{n}}\right) \\
r_{n}=2^{2.8^{n-1}}, \lambda_{n}=8 r_{n}^{3 / 2} .
\end{gathered}
$$

Define

$$
f(z)=f_{1}(z) f_{2}(z)
$$

Then $f(z)$ is an entire function of order $3 / 2$ for which $\delta(0)>0$ see A. A. Goldberg [1].

Thus 0 is an e.v.N for $f(z)$, so $\mu(r, f) \rightarrow 0$ by the lemma. But 0 is not an asymptotic value for $f(z)$.

3. THEOREM 2. Let $F(z)$ be a meromorphic function of order $\rho(0<\rho<\infty)$; and let $\rho(r)$ be Lindelöf proximate order relative to $T(r, F)$. Let $n\left(r, a_{i}\right)$ be the number of zeros of $F(z)-a_{i}$ in $|z| \leqq r$; all the $a_{i}$ being different $\left(0 \leqq\left|a_{i}\right|\right.$ $\leqq \infty$; in case $a_{i}=\infty, n\left(r, a_{i}\right)=n(r, \infty)$ is the number of poles $)$. Then

$$
\lim _{r \rightarrow \infty} \sup \sum_{i=1}^{q} \frac{n\left(r, a_{i}\right)}{r^{\rho(r)}} \geqq(q-2) \rho
$$

where $q$ is an integer $\geqq 3$.

PROOF. Let $\quad \limsup _{r \rightarrow \infty} \sum_{i=1}^{q} \frac{n\left(r, a_{i}\right)}{r^{\rho(r)}}=k$ 
and if possible let $k<(q-2) \rho$, then

$$
\sum_{i=1}^{q} n\left(r, a_{i}\right)<(k+\varepsilon) r^{\rho(r)} \quad \text { for } r \geqq r_{0} .
$$

Hence

$$
\begin{aligned}
\sum_{i=1}^{q} N\left(r, a_{i}\right) & +O(\log r)<(k+\varepsilon) \int_{r_{0}}^{r} t^{\rho(t)-1} d t \sim \frac{k+\varepsilon}{\rho} r^{\rho(r)} \\
& =\frac{k+\varepsilon}{\rho} T(r, F)
\end{aligned}
$$

for a sequence of values of $r$.

Further from the second theorem of Nevanlinna

$$
(q-2) T(r, F)<\sum_{i=1}^{q} N\left(r, a_{i}\right)+O(\log r) .
$$

Hence for an infinity of values of $r$ we have $(q-2) T(r, F)<\frac{k+\varepsilon}{\rho} T(r, F)+O(\log r)$, and since $\varepsilon$ is arbitrarily small $(q-2) \rho \leqq k$. This gives a contradiction. Hence the result follows.

4. Let $f(z)$ be an entire function and let $\rho_{1}(a)$ be the exponent of convergence of the zeros of $f(z)-a$. If $\rho_{1}(a)<\rho$ we say that $a$ is an e.v. B for $f(z)$.

If

$$
\lim _{r \rightarrow \infty} \inf \frac{T(r, f)}{n(r, a) \phi(r)}>0
$$

for a positive non-decreasing function $\phi(x)$ such that

$$
\int_{A}^{\infty} \frac{d x}{x \phi(x)}<\infty
$$

then $a$ is defined to be an $\mathrm{e} \cdot \mathrm{v} \cdot \mathrm{E}$, see S.M.Shah [2]. Let $f(z)$ be an entire function of order $\rho(0<\rho<\infty)$ and let $\rho(r)$ be proximate order relative to $\log M(r, f)$, that is,

$$
\begin{aligned}
& \rho(r) \rightarrow \rho \text { as } r \rightarrow \infty, \\
& r \rho^{\prime}(r) \log r \rightarrow 0 \text { as } r \rightarrow \infty, \\
& \log M(r, f) \leqq r^{\rho(r)} \text { for } r \geqq r_{0}
\end{aligned}
$$

and $\log M(r, f)=r^{\rho(r)}$ for a sequence of values of $r$. Valiron has proved that for a class of entire functions of finite non-zero order if 


$$
\frac{n(r, a)}{r^{\rho(r)}} \rightarrow 0 \text { as } r \rightarrow \infty
$$

then $\frac{n(r, x)}{r^{\rho(r)}}$ lies between two positive constants for every $x \neq a$.

Hence it is reasonable to define $a$ as an exceptional value in some sense if

$$
\lim _{r \rightarrow \infty} \frac{n(r, a)}{r^{p(r)}}=0
$$

We shall call $a$ as an e.v.L(in the sense of Lindelöf) if (1) holds.

THEOREN 3. (i) If $a$ is an e.v.B then $a$ is an e.v.L also but the converse is not true.

(ii) If $a$ is an e.v.L then $a$ is e.v. $V$ with $\Delta(a)=1$.

(iii) If $a$ is e.v.E then $a$ is e.v.L also but the converse is not true.

PROOF. (i) Let $a$ be e.v. B then

so

$$
n(r, a)=O\left(r^{c}\right) \quad c<\rho
$$

$\frac{n(r, a)}{r^{\rho(r)}}<A r^{c-\rho(r)} \quad$ for $r \geqq r_{0}$.

Further $\quad \rho(r)>\frac{\rho+c}{2} \quad$ for $r \geqq r_{0}$.

Hence $\quad \frac{n(r, a)}{r^{\rho(r)}} \rightarrow 0$ as $r \rightarrow \infty$

so $a$ is e.v. L.

That the converse is not true can be seen from the function

$$
f(z)=\prod_{2}^{\infty}\left(1+\frac{z}{n(\log n)^{2}}\right)
$$

Here

$$
M(r, f) \sim \frac{r}{\log r}, \quad n(r, 0) \sim \frac{r}{(\log r)^{2}}
$$

Set

$$
\rho(r)=1-\frac{\log \log r}{\log r}
$$

Then it can easily be seen that $\rho(r)$ is a proximate order relative to $\log M(r, f)$. 
ON EXCEPTIONAL VALUES OF ENTIRE AND MEROMORPHIC FUNCTIONS 377

Here

$$
\rho=\rho_{1}(0)=1,
$$

so 0 is not e.v. B.

But

$$
\frac{n(r, 0)}{r^{\rho(r)}} \rightarrow 0 \text { as } r \rightarrow \infty
$$

and $\frac{n(r, x)}{r^{\rho(r)}}$ lies between two positive constants for all $x \neq 0$ and thus 0 is e.v.L.

(ii) Let $a$ be e.v.L then

$$
\begin{aligned}
& n(r, a)<\varepsilon r^{\rho(r)} \text { for } r \geqq r_{0}, \\
& N(r, a)<\varepsilon \int_{r_{0}}^{r} t^{\rho(t)-1} d t \sim \frac{\varepsilon r^{\rho(r)}}{\rho}, \\
N(2 r, a) \quad & \frac{\varepsilon}{\rho}(2 r)^{\rho(2(r)} \sim \frac{\varepsilon}{\rho} 2^{\rho} r^{\rho(r)} \\
= & \frac{\varepsilon}{\rho} 2^{\rho} \log M(r, f) \text { for a sequence of values of } r \\
< & \frac{\varepsilon}{\rho} 2^{\rho} T(2 r, f) . \\
\text { Hence } \quad & \lim _{r \rightarrow \infty} \inf \frac{N(r, a)}{T(r, f)}=0 .
\end{aligned}
$$

So $a$ is $\mathrm{e} \cdot \mathrm{v} \cdot \mathrm{V}$ with $\Delta(a)=1$.

We omit the proof of the first part of (iii). That the converse is not true can again be seen from the same example

$$
f(z)=\prod_{2}^{\infty}\left(1+\frac{z}{n(\log n)^{2}}\right) .
$$

Here $\quad \frac{\log M(r, f)}{n(r, 0) \phi(r)} \sim \frac{\frac{r}{\log r}}{\frac{r}{(\log r)^{2}} \phi(r)}=\frac{\log r}{\phi(r)}$. 
But since $\quad \int_{\Delta}^{\infty} \frac{d x}{x \phi(x)}<\infty$ so $\log x=o(\phi(x))$ when $x \rightarrow \infty$.

Hence $\quad \lim _{r \rightarrow \infty} \frac{\log M(r, f)}{n(r, 0) \phi(r)}=0$.

Hence a fortiori

$$
\lim _{r \rightarrow \infty} \frac{T(r, f)}{n(r, 0) \phi(r)}=0
$$

So 0 is not e.v.E though it is e.v. L.

5. Nevanlinna [3] has proved that if $F(z)$ is a meromorphic function of order $\rho<1 / 2$ for which

$$
\lim _{r \rightarrow \infty} \frac{N(r, a)}{T(r, F)}=0
$$

then $\quad \limsup _{r \rightarrow \infty} \frac{N(r, x)}{T(r, F)}=1$

for every $x \neq a(0 \leqq|x| \leqq \infty)$.

Of course (3) is not true for every meromorphic function of order $<1 / 2$ for every $x$. For instance if $f(z)$ is an entire function of order $<1 / 2$ then $F(z)=$ $f(z) /(z-a)$ will be a meromorphic function of order $<1 / 2$ for which

$$
\frac{N(r, \infty)}{T(r, F)} \rightarrow 0 \text { as } r \rightarrow \infty
$$

We give a method to construct a class of meromorphic functions of any given order for which (3) holds for every $x(0 \leqq|x| \leqq \infty)$. We prove :

THEOREM 4. Given any $\rho(0<\rho<\infty)$, there exists a meromorphic function of order $\rho$ for which (3) holds for every $x(0 \leqq|x| \leqq \infty)$.

Let $f(z)=\sum_{n=0}^{\infty} a_{n} z^{\lambda_{n}}$ be an entire function of order $\rho$ for which 


$$
\sum_{n=0}^{\infty} \frac{1}{\lambda_{n+1}-\lambda_{n}}<\infty
$$

and let as usual

$$
\mu(r, f)=\operatorname{Min}_{|z|=r}|f(z)|,
$$

then $\mu(r, f) \rightarrow \infty$ as $r \rightarrow \infty$ through a sequence $r=r_{n}$; see A. J. Macintyre and P. Erdös [4].

Set

$$
F(z)=\frac{1}{f(z)}+z
$$

then $T(r, F)=T(r, f)+O(\log r)$.

Let $a$ be any number such that $0 \leqq|a|<\infty$. Then for $|z|=r_{n}$ we have uniformly as $n \rightarrow \infty$

$$
F(z)-a=z-a+o(1)
$$

Hence

$$
m\left(r, \frac{1}{F-a}\right)=\frac{1}{2 \pi} \int_{0}^{2 \pi} \log ^{+}\left|\frac{1}{F-a}\right| d \theta=0
$$

for $r=r_{n}$ and $n>n_{0}$.

Hence $\quad \lim _{r \rightarrow \infty} \sup \frac{N(r, F-a)}{T(r, F)}=1$.

Also $m(r, F)=\log r+o(1)=o(T(r, F))$ as $r \rightarrow \infty$ through the sequence $r=r_{n}$. Hence

$$
\limsup _{r \rightarrow \infty} \frac{N(r, F)}{T(r, F)}=1
$$

This proves (3) for every finite or infinite $x$.

REMARK. If the meromorphic function is of order $<1 / 2$, the construction is still easier, since in (4) any entire function $f(z)$ of order $<1 / 2$ will serve the purpose, because by a well known theorem of Wiman for such an entire function $\lim _{r \rightarrow \infty} \sup \mu(r, f)=\infty$. We also remark that by choosing a suitable entire function 
$f(z)$ for which the sequence $r_{n}$ is sufficiently dense we could have even achieved

for every $x(0 \leqq|x| \leqq \infty)$.

$$
\lim _{r \rightarrow \infty} \frac{N(r, x)}{T(r, F)}=1
$$

Finally we prove:

THEOREM 5. For every meromorphic function $F(z)$ of order $\rho(0 \leqq \rho<\infty)$,

$$
\limsup _{r \rightarrow \infty} \frac{n(r, a)}{T(r, F)} \geqq \rho
$$

provided that a is not e.v. $V$ for $F(z)$.

We omit the proof.

I take this opportunity to thank Professor W. K. Hayman for his valuable suggestions.

\section{REFERENCES}

[1] A. A. GoldBerG, An example of an entire function of finite order with a nonasymptotic defective value (Russian), Uzgorod Gos. Univ. Naucn. Zap. 18(1957) 191-194. (see also Math. Review 20, No.1(1959), Review no. 111).

[2] S. M. SHAH, Exceptional values of entire and meromorphic functions, Duke Math.J., 19(1952), 585-594.

[3] R. Nevanlinna, Le théorème de Picard-Borel et la théorie des fonctions méromorphes, Paris, 1929, p. 55.

[4] A. J. MAcintYre and P. ERDös, Integral functions with gap power series, Proc. Edin. Math. Soc., Series 2, 10, Part II(1954),62-70. 